\title{
Applying principles of behaviour change to reduce SARS-CoV-2 transmission
}

\author{
Robert West $\mathbb{1}^{1 凶}$, Susan Michie ${ }^{\circledR}{ }^{2}$, G. James Rubin ${ }^{3}$ and Richard Amlôt ${ }^{4}$
}

\begin{abstract}
Human behaviour is central to transmission of SARS-Cov-2, the virus that causes COVID-19, and changing behaviour is crucial to preventing transmission in the absence of pharmaceutical interventions. Isolation and social distancing measures, including edicts to stay at home, have been brought into place across the globe to reduce transmission of the virus, but at a huge cost to individuals and society. In addition to these measures, we urgently need effective interventions to increase adherence to behaviours that individuals in communities can enact to protect themselves and others: use of tissues to catch expelled droplets from coughs or sneezes, use of face masks as appropriate, hand-washing on all occasions when required, disinfecting objects and surfaces, physical distancing, and not touching one's eyes, nose or mouth. There is an urgent need for direct evidence to inform development of such interventions, but it is possible to make a start by applying behavioural science methods and models.
\end{abstract}

T here are many actors whose behaviour is crucial to limiting the transmission of the SARS-CoV-2 virus, which causes COVID-19. These include governments, health and social care organisations, businesses, media outlets and community groups. Decisions they make have far-reaching effects on virus transmission itself and also on the cost to people and society, both of the disease and of the measures taken to control it. These actors form part of a complex, interacting system that needs to be mapped and understood ${ }^{1}$. At the heart of the system are individual members of communities and their behaviours that are transmitting the virus.

The behavioural sciences seek to understand the psychological, biological, social and environmental factors that influence behaviour with a view to developing interventions and policies to help achieve societal, organisational or personal goals. When it comes to limiting SARS-CoV-2 transmission, they are already being brought to bear on the problem ${ }^{2-5}$. They can inform policies to (i) control the infection, (ii) mitigate the harm done by it and the measures taken to control it, and (iii) develop resilience and new patterns of behaviour in preparation for future pandemics ${ }^{5,6}$. All this is in addition to the very important role that clinical, psychological and social sciences can play in addressing the impact of COVID-19 on mental health and societal functioning ${ }^{6,7}$.

This paper focuses on adherence to behaviours required to reduce virus transmission. We argue that there is an urgent need to develop and evaluate interventions to promote effective enactment of these behaviours and provides a preliminary analysis to help guide this. This is relevant for the current phase of the pandemic and to reduce the risk of resurgence in months to come and of future pandemics.

\section{Behaviours required to reduce SARS-CoV-2 transmission} COVID-19 is the disease caused by the SARS-CoV-2 virus, which is a novel form of coronavirus ${ }^{8}$. The virus is transmitted in communities either directly by travelling through the air from an infected person's airways, mouth or nose to a recipient's eyes, nose or mouth (the T-zone), or by the virus contaminating an object or surface (fomite) that is touched by a recipient who then goes on to touch their T-zone? . The T-zone is the primary route for the virus to cause infection because the virus enters cells of mucous membranes and lung epithelial tissue ${ }^{8}$. It does not enter through the skin. The evidence suggests that, in community settings, the virus is carried primarily on respiratory droplets (relatively large particles that typically travel a short distance before falling to a surface) rather than aerosol (small particles than can stay airborne for an extended period) ${ }^{10,11}$, but this view has been contested ${ }^{12}$. Figure 1 shows the putative transmission paths and the behaviours that can block them in community (as opposed to healthcares) settings.

Governments have mainly used isolation (keeping vulnerable and infected or potentially infected people physically away from others) and what has been termed 'social distancing' (staying at home except for essential journeys) to block transmission. These are represented by the blue vertical bar in Fig. 1, which separates the infected person and fomites on the left of the diagram from other people on the right. Measures taken to ensure social distancing have included closing all but essential shops and businesses, banning gatherings and instructing people to stay at home except for essential journeys and work.

Isolation and social distancing measures appear to be effective in controlling the pandemic ${ }^{13}$. Unfortunately they come at an enormous cost to people's livelihoods, education and mental health, as well as to the global economy ${ }^{14}$. Adherence to isolation and social distancing behaviours faces strong practical, motivational and social barriers and also imposes considerable costs on people and society. These costs are borne disproportionately by people who are already disadvantaged ${ }^{15}$. Moreover, until and unless an effective vaccine can be found, a resurgence of infection is likely when these measures are relaxed. Widespread and rigorous adherence to 'personal protective behaviours' (individual behaviours aimed at protecting oneself and others) as set out in Fig. 1 is required. These behaviours are also needed to protect people who have to put themselves at risk of catching the disease in the course of vital functions they perform in society.

'Department of Behavioural Science and Health, Institute of Epidemiology and Healthcare, University College London, London, UK. ${ }^{2}$ Research Department of Clinical, Educational and Health Psychology, University College London, London, UK. 'Department of Psychological Medicine, King's College London, London, UK. ${ }^{4}$ Behavioural Science Team, Emergency Response Department Science and Technology (ERD S\&T), Public Health England, Salisbury, UK.

凶e-mail: robert.west@ucl.ac.uk 


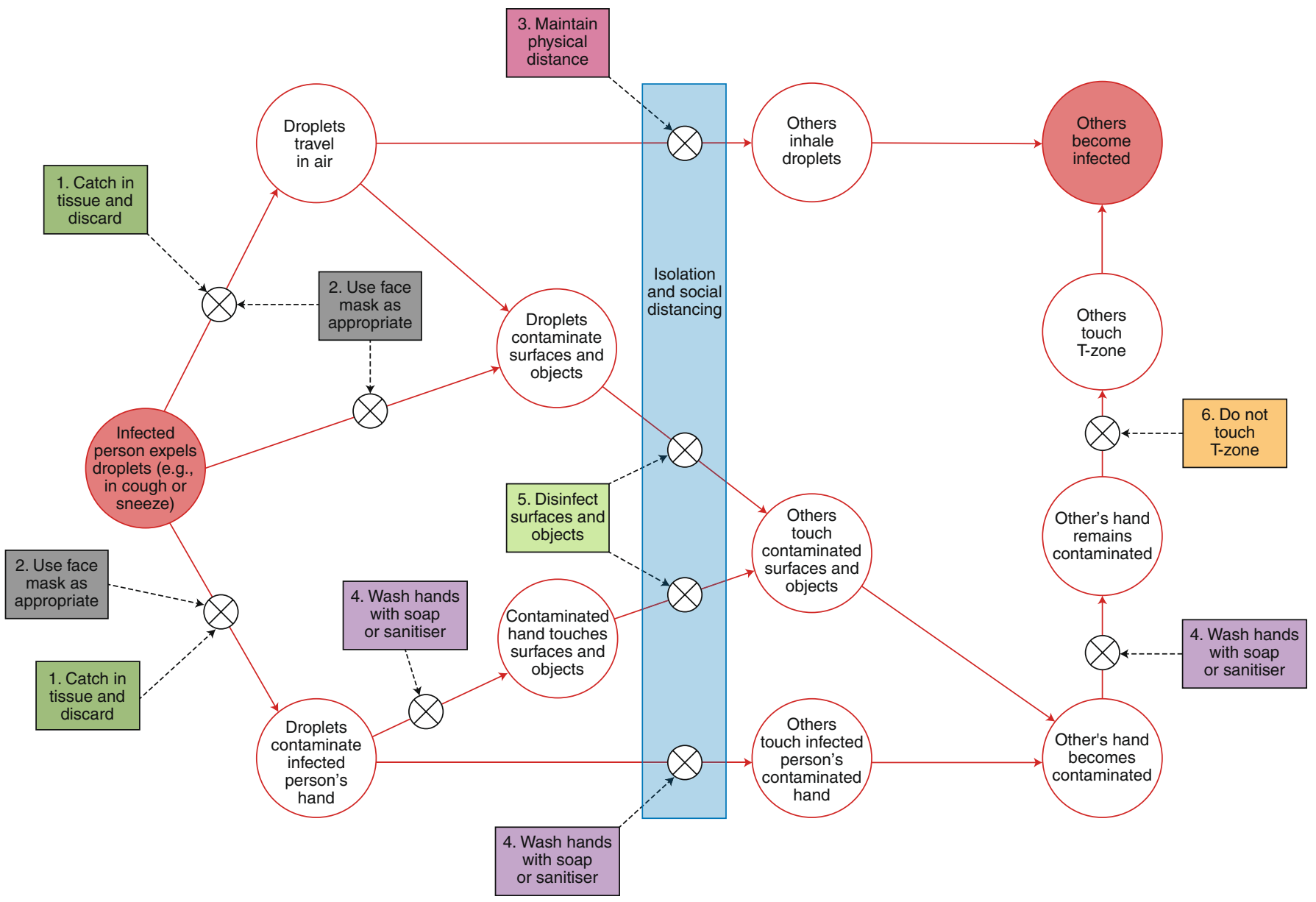

Fig. 1| Pathways to SARS-CoV-2 transmission and behaviours to block this. Large circles, stages in the pathway; red arrows, routes of transmission; crosses in small circles, blocks to transmission; filled rectangles, personal protective behaviours to block transmission routes. Dotted arrows point to the blocking points. The blue vertical bar shows the points at which isolation and social distancing measures work. Reproduced from ref. ${ }^{47}$.

The biomechanics of transmission mean that it could be reduced at source by the infected person making sure that they cough or sneeze into a tissue that is immediately disposed of in a way that does not cause further contamination (Box 1 in Fig. 1). Face masks (Box 2 in Fig. 1) can act as a physical barrier to the spread of droplets, but their effectiveness in reducing virus transmission may be offset by people failing to use them appropriately, and what limited evidence is available as of the end of April 2020 has not clearly shown a benefit in community settings ${ }^{16}$.

Physical distancing (Box 3 in Fig. 1) aims to minimise the risk of direct transmission of the virus via inhaled droplets. The distance at which people are thought to be at risk from direct inhalation of contaminated droplets in normal circumstances is currently estimated at up to $2 \mathrm{~m}$ (ref. ${ }^{17}$ ). However, it has been suggested that under certain conditions droplets or aerosol may travel further than this ${ }^{18}$.

Given the importance of fomites in transmission of the virus ${ }^{19}$, washing hands with soap or hand sanitiser (Box 4 in Fig. 1) and disinfecting objects and surfaces (fomites) (Box 5 in Fig. 1) may substantially reduce transmission. Transmission by fomites occurs because people touch their T-zone after touching them. The fomite route to transmission of the virus could be particularly important because the virus can survive on some surfaces for several days ${ }^{20}$. Therefore, not touching the T-zone (Box 6 in Fig. 1) may be an important behaviour to target. Although the above personal protective behaviours are included in government advice in a number of countries $^{21-23}$, little guidance, training or support is given to promote adherence, even though failure to do so is critical to the transmission of the virus.

\section{Understanding behaviour and how to change it}

Ideally we would be able to draw on high-quality intervention evaluations to identify ways to increase enactment of personal protective behaviours. Unfortunately, there is a dearth of studies on this. There are some suggestions on how best to promote adherence to social distancing, for example ${ }^{5}$. While a considerable amount of research has been undertaken on hand-hygiene in certain settings ${ }^{24-26}$, generalisability to community settings is limited. We could find no published evaluations of interventions to reduce T-zone touching. There is some research on psychological interventions to reduce itch scratching in people suffering from atopic dermatitis, but no firm conclusions have been reached ${ }^{27,28}$.

In the absence of strong direct evidence to guide interventions, we can draw on behaviour-change principles to generate ideas as to what strategies to adopt. A staged process has been proposed for doing this, known as the 'behaviour change wheel' ${ }^{29-31}$. This was derived from a synthesis of 19 major behaviour-change frameworks. It starts with an analysis of the capability, opportunity and motivation required to enact each behaviour. This is followed by mapping these requirements to relevant types of intervention (education, persuasion, incentivization, coercion, training, restriction, environmental restructuring, modelling and enablement) and types 


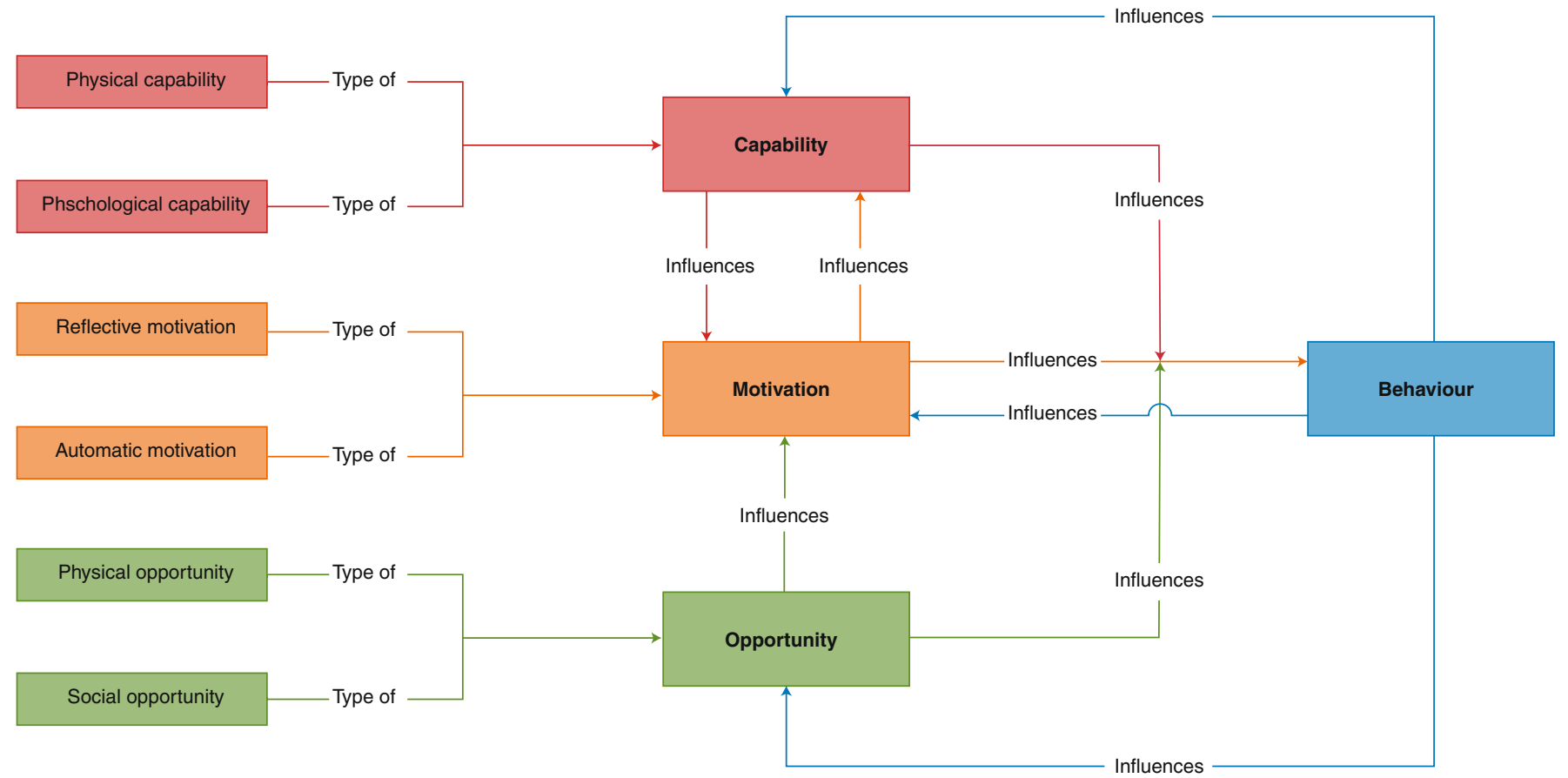

Fig. 2 | The capability-opportunity-motivation-behaviour (COM-B) model. Reproduced from ref. ${ }^{48}$. Capability is an attribute of a person that together with opportunity makes a behaviour possible or facilitates it. Opportunity is an attribute of an environmental system that together with capability makes a behaviour possible or facilitates it. Motivation is an aggregate of mental processes that energise and direct behaviour. Behaviour is individual human activity that involves co-ordinated contraction of striated muscles controlled by the brain. Physical capability is capability that involves a person's physique and musculoskeletal functioning (e.g., balance and dexterity). Psychological capability is capability that involves a person's mental functioning (e.g., understanding and memory). Reflective motivation is motivation that involves conscious thought processes (e.g., plans and evaluations). Automatic motivation is motivation that involves habitual, instinctive, drive-related and affective processes (e.g., desires and habits). Physical opportunity is opportunity that involves inanimate parts of the environmental system and time (e.g., financial and material resources). Social opportunity is opportunity that involves other people and organisations (e.g., culture and social norms).

of policies best suited to delivering these (communications and marketing, guidelines, service provision, fiscal measures, regulation, legislation, environmental and social planning). This can then form a basis for a detailed specification of an intervention strategy and its proposed implementation, including the specific behaviour change techniques to use ${ }^{32}$. Throughout this process it is important to evaluate the options in terms of acceptability, practicability, effectiveness, affordability, spill-over effects, and equity (APEASE criteria $^{29,31}$. All available evidence, direct and indirect, should be brought to bear on this evaluation.

Thus, the first stage in the process involves undertaking what has been termed a 'behavioural diagnosis' using the capability, opportunity, motivation and behaviour (COM-B) model $^{29,30}$. Figure 2 shows a formal representation of the model, outlining how the different components relate to each other.

A principal tenet of the model is the common-sense idea that, at any given time, a behaviour occurs when both the capability and opportunity are present and when the person is more motivated to enact that behaviour than any other. A second key tenet is that capability, motivation, opportunity and behaviour are causally linked to each other in feedback loops, so that increasing capability or opportunity can influence motivation, and that behaviours feed back to influence opportunity, capability and motivation.

The motivational part of COM-B is elaborated in the PRIME theory of motivation ${ }^{33}$ (Fig. 3). This recognises that any behaviour can be influenced by both reflective and automatic processes. It proposes that these do not operate in parallel but rather that the proximal cause of all behaviour is always the balance between potentially competing impulses and inhibitions.
Potentially competing impulses and inhibitions are controlled by instinct and habit processes, plus any motives (wants or needs) that are present at the time. Wants and needs are generated by feelings of anticipated pleasure or satisfaction and of anticipated relief from discomfort or drive states. All of this makes up our 'automatic' motivation.

As humans we have the capacity to think about what we do and make conscious decisions-what may be termed 'reflective motivation'. So, apart from wants and needs, there are thought processes that create and compare evaluations: beliefs about what is beneficial or harmful and right or wrong. These processes underlie our conscious decision-making, when we weigh up the costs and benefits of courses of action or work out solutions to problems. We also have the capacity to plan ahead, and these plans form much of the structure of our behaviour over the course of minutes, hours, days, weeks and years.

PRIME theory also recognises the importance of identity: the aggregate of our beliefs and the images of ourselves as we are, have been or could be, with the feelings attached to these ${ }^{34}$. These can be so strong that they even override need for survival. Finally, PRIME theory recognises the importance of imitation and modelling in human behaviour $^{35}$. This occurs at the level of small-scale behaviours and mannerisms (for example, face touching) as well as much larger-scale behaviours, evaluations and wants and needs. It should also be noted that wants and needs influence evaluations. A well-recognised example is what has been termed 'wishful thinking' ${ }^{36}$.

PRIME theory is not a replacement for more specific models that focus on particular motivational processes, but rather a way of linking them together. Of the more specific models, many are rel- 


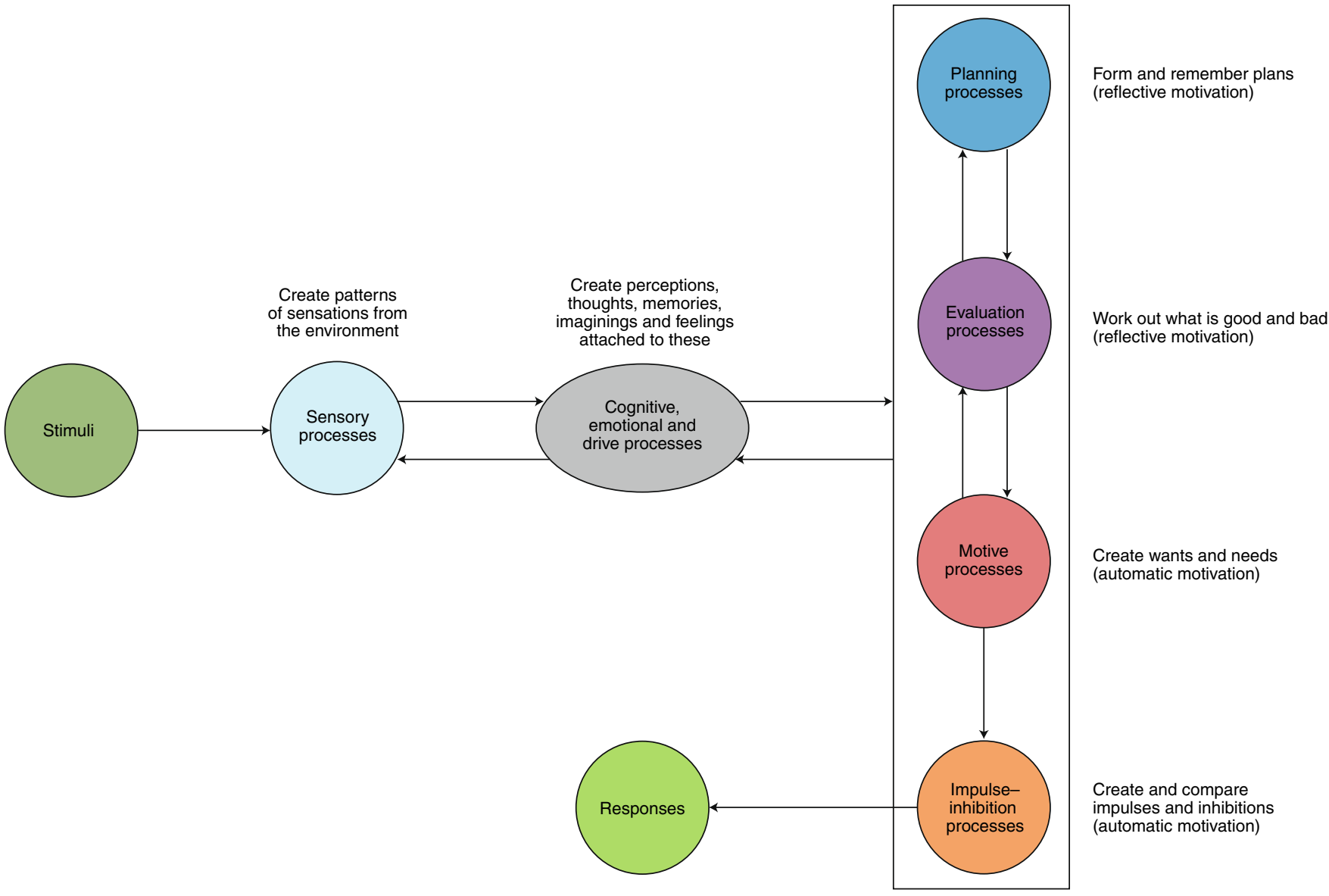

Fig. 3 | The processes involved in human motivation according to PRIME theory. Arrows denote the 'influence' relationship. Reproduced from ref. ${ }^{48}$.

evant to understanding behaviours that can block the transmission of SARS-CoV-2. A compendium of behaviour-change models has been put together that can help identify ones that may be relevant in any given situation ${ }^{37}$. These can be supplemented by models that focus on conscious decision-making processes (for example, ref. ${ }^{38}$ ), on emotional and habit processes (for example, ref. ${ }^{39}$ ) or on environmental influences (for example, ref. ${ }^{40}$ ).

The main tenets of PRIME theory and examples of more specific models are summarised in Table 1. There are many others that are potentially relevant, but those chosen cover key principles to consider. Table 1 also gives examples of possible implications of the models for interventions to promote personal protective behaviours.

The principles described in Table 1 can help us begin to map out what is required in terms of capability, opportunity and motivation for each of the behaviours identified in Fig. 1. Table 2 provides a preliminary behavioural diagnosis for each of the protective behaviours, taking as read that they will all be enabled by having a 'mental model' of transmission routes and the ways in which the behaviours block these routes ${ }^{41}$.

In general, the capability to undertake personal protective behaviours requires people to understand what needs to be done, under what precise circumstances it needs to be done, how to do it and why it is important. It also requires development of appropriate skills and techniques. Opportunity requires ensuring access to the living and working arrangements, tools and resources that enable the behaviours to be enacted and, crucially, to maintain normal life as far as possible despite these behaviours. It also involves creating the social opportunity to support the behaviours, including norms and social rules. Motivation involves, at a minimum, people feeling a strong need to enact the behaviours in all the circumstances in which they are required, and this must be sufficient to overcome competing wants or needs in the moment. People should see the behaviour being enacted as valued within the group or groups they identify with and see other people enacting them. People need to develop rules and habits to sustain the behaviour. There will be large differences between people and between groups of people (for example, by personality, age, employment status and type of neighbourhood) in their capability, opportunity and motivation.

\section{Interventions to promote behaviours to limit virus transmission}

As noted earlier, the behaviour change wheel sets out nine broad categories of intervention that can be included in any behaviour change strategy: education, persuasion, incentivization, coercion, enablement, training, restriction, environmental restructuring and modelling ${ }^{29-31}$. It also specifies criteria for evaluating intervention options. Table 3 summarises these criteria. Thus an intervention may be likely to be effective but to have unacceptable spill-over effects, or it may be impracticable or unacceptable to key stakeholders. An example of applying this approach to reducing COVID-19 transmission can be seen in a behavioural science paper presented to UK's Scientific Advisory Group in Emergencies outlining intervention options for increasing adherence to social distancing measures and consideration of them using the APEASE criteria ${ }^{42}$.

We applied the principles in Table 1 to the behaviours in Table 2 and evaluated these using criteria in Table 3. This led to a set of illustrative recommendations, as set out in Table 4. It must be emphasised that this is a preliminary analysis, and for formal recommendations, a much fuller and more systematic analysis would 


\section{Table 1 | Models of behaviour focusing on different motivational processes}

Model Brief summary

PRIME theory ${ }^{33}$

Prospect theory of judgement and decision-making; judgement heuristics ${ }^{38}$

Conflict

theory of decision making ${ }^{49}$

Cognitive dissonance theory ${ }^{50}$

Temporal discounting ${ }^{5}$

Social norm theory ${ }^{40}$

Operant learning theory ${ }^{39}$

Habit theory ${ }^{44}$ imagined. functions.
Behaviour at any one moment results from the strongest of potentially competing impulses and inhibitions operating at that moment. These are driven by habit and instinct processes as well as by feelings of want or need. Wants and needs in turn are driven by emotions and drive processes as well as by evaluations. Evaluations are driven by judgement processes, wants and needs and plans. Plans are created by judgement processes and enacted when they are recalled and when they generate sufficiently strong wants and needs, and then generate impulses or inhibitions to overcome others that are being generated by the immediate situation. Identity and modelling play an important role in the whole motivational system.

Focuses on evaluations: We make decisions based on comparison of the positive and negative consequences of options under consideration, weighted by the subjective likelihood of those consequences occurring. Our evaluations and subjective likelihoods are subject to important biases, including (i) overweighting negative outcomes compared with positive ones, (ii) overweighting certainty compared with near certainty, (iii) underweighting numerical differences in value against a background of large numbers compared with small numbers and (iv) judging outcomes to be more likely if they are more readily

Focuses on evaluations and plans: Our decision-making processes are radically altered by the conditions under which the decision takes place, and particularly the degree of stress involved. 'Vigilant decision-making', the optimal mode, gives way to suboptimal modes of decision-making under defined conditions: (i) unconflicted inertia when the risks of inaction are not judged to be serious, (ii) unconflicted change when risks of action are seen as minimal, (iii) defensive avoidance when there seems little hope of avoiding negative outcomes, and (iv) hypervigilance when there is strong time pressure and some hope of finding a solution. Following the decision, suboptimal modes of decision-making lead to defective strategies for coping with negative outcomes, whether or not these were avoidable.

Focuses on evaluations: We experience negative emotions when we notice that we have beliefs that conflict with each other, and we feel a need to reduce these by changing our beliefs, suppressing them or adding new beliefs.

Focuses on evaluations: When making decisions, we undervalue outcomes to a greater extent the further they are expected to occur in the future. People differ with regard to the shape of the temporal discounting curve, with people who are susceptible to impulse control problems tending to have steeper discounting

Focuses on evaluations and wants and needs: People adopt values, motives and behaviours that they perceive as normative within groups with which they identify.

Focuses on wants and needs and impulses: Through a process of associative learning, we learn to want to enact, and experience impulses to engage in, behaviours that are contingently followed by positive emotional experiences or relief from unpleasant ones or from drive states, even when we are not consciously aware of the associations.

Focuses on impulses: Repetition of behaviours in a given context results in those behaviours becoming increasingly automatized, occurring without conscious thought.
Examples of insights relevant to behaviours to limit transmission of SARS-CoV-2

Messaging to the public should aim to create a strongly felt 'need' to engage in protective behaviours rather than just a belief that one 'should' do them. People should be supported to develop plans that are specific and strongly linked to identity through development of personal rules (for example, always washing hands when entering one's home). Interventions should recognise the balance of impulses and inhibitions at key moments, and promote development of habits that come into play when needed. Educational materials should include modelling of desired behaviours.

Interventions need to ensure that perceived benefits of protective actions outweigh the costs, and support should be provided to mitigate the costs. Benefits should be framed in terms of certainties and avoidance of negative outcomes, and they should be made readily imaginable.

Messaging and support should create 'concern' that motivates action rather than anxiety that could lead to defensive avoidance. This involves providing a clear indication of practical and realistic steps that can be taken to address the risk with a strong sense that these will work.

Messaging to the public should aim to heighten dissonance linked to nonadherence, and it should aim to prevent people engaging in 'exceptionalism', where they add beliefs about their situation being a special case that means they do not have to adhere.

Communications promoting protective behaviours should aim to bring the benefits into people's immediate time horizon, and any use of incentives and or punishments should focus on creating immediate contingencies.

Interventions, including communications, should maximise the visibility and approval of desired behaviours and should minimise the visibility of undesired ones by groups with which the target groups identify.

Social rewards, for example, through praise, should be liberally used to maintain desired behaviours, and people should be encouraged to support each other in this way. Where sanctions are used, it is important for these to be consistently applied.

Resources supporting protective behaviours should help people to identify and train the required habits, including habits that conflict with behaviours one is attempting to prevent. 
Table 1 | Models of behaviour focusing on different motivational processes (Continued)

Model

Brief summary

\section{Examples of insights relevant to behaviours to limit} transmission of SARS-CoV-2

Five-factor

theory of

personality ${ }^{52}$
Covers all motivational processes: People range along five broad continua in terms of emotional reactions, wants and needs, and ways of thinking: (i) extraversion-introversion, with extraverts tending to find social stimuli more rewarding, (ii) stabilityneuroticism, with more stable people tending to feel less anxious and threatened by events, (iii) openness versus closed-mindednes, with open-minded people tending to be curious and independent thinkers, (iv) conscientiousness-carelessness, with conscientious people being more thorough and careful and less impulsive, and (v) agreeableness-disagreeableness, with more agreeable people tending to be helpful, trusting and empathetic.
Messaging, regulatory and enabling interventions should recognise individual differences in factors influencing protective behaviours; for example, some people will require more stringent measures than others, and level of concern will need to be raised for some people while anxiety will need to be reduced , for others. . 
Table 2 | Behaviours required to limit SARS-CoV-2 transmission and what is required for these to be enacted (Continued)

\begin{tabular}{l} 
Behaviour \\
\hline Social distancing \\
(staying at home \\
except under \\
specifically \\
defined \\
circumstances)
\end{tabular}

\section{Capability}

Understanding the importance of this and ways of mitigating the adverse consequences, both physical and psychological. Understanding the precise circumstances in which people can leave their home and where they can go.

Isolation of people who have symptoms or who are highly vulnerable
Understanding the importance of this and ways of achieving it tailored to the specific household and family circumstances. Understanding ways of mitigating the adverse psychological effects.

\section{Opportunity}

Availability of services and resources to maintain livelihood and physical and mental health. Access to safe facilities for shopping and exercise on the occasions when these are permitted. Access to home-based leisure facilities and ways of carrying on working that do not require going out. Organisational rules that permit working at home or time off.

Access to space and physical domestic arrangements that make this possible. Access to services and resources to iccess sustain the behaviour and be motivated to to home-based leisure facilities and ways of find ways to overcome specific challenges carrying on working that can be undertaken that occur from time to time. in isolation.

\section{Motivation}

People must feel a very strong need to stay at home and obey social distancing rules, and this must be stronger at all times than the want or need to socialise, earn money or pursue usual activities.

People must feel a need to maintain isolation in the face of practical and ind ways to overcome specific challenges (2)

\section{Table 3 | APEASE criteria for evaluating intervention approaches or components}

\begin{tabular}{ll} 
Criterion & To what extent ... \\
\hline Acceptability & is it judged to be acceptable by all key stakeholders \\
\hline Practicability & can it be delivered as intended, at the scale intended and in the context intended \\
Effectiveness & will it deliver the desired outcome in the target population \\
Affordability & can it be afforded within an acceptable budget \\
Spill-over effects & is it likely to have additional negative or positive consequences \\
Equity & is it likely to increase or decrease inequalities in society
\end{tabular}

be required using the principles set out in the behaviour change wheel guide $\mathrm{e}^{29,31}$.

Based on this preliminary analysis, different personal protective behaviours appear to require different types of intervention. Education is important, but unlikely to be sufficient. Persuasion and modelling will likely be crucial in motivating people to enact many of the behaviours. Use of social incentives and supportive and carefully applied coercive interventions will also presumably be important in some cases. Less obviously, training and various forms of enablement will likely be required to support some of the behaviours. Environmental restructuring will probably have a crucial role to play, in terms of the physical environment, provision of financial and material resources, and ensuring that social rules and norms are supportive of the required behaviours. It seems likely that mass media, social media and online platforms will be key to delivering interventions to promote personal protective behaviours. These will probably need to be supplemented by service provision by key workers in certain cases. Regulation and environmental planning may be needed in some cases, as will legislation.

Of the personal protective behaviours, one that may merit particular attention is not touching the T-zone. This is because the potential impact may be high if it can be achieved, it requires no additional facilities and appears to have no negative spill-over effects. The key question is whether effective interventions can be found to achieve this ${ }^{43}$. Combating the habit element could, for example, involve training conflicting habits (such as keeping hands below shoulder level), creating physical or behavioural barriers, or generating mindfulness to bring the behaviour to awareness before it is completed ${ }^{43,44}$. Training people not to touch their T-zone could be delivered, for example, by an online application using artificial intelligence to provide feedback on behaviours detected by webcams. Tackling what is probably the other primary driver, a feeling of need to scratch an itch, could involve techniques falling under the acronym DEADS: delay, escape, avoid, distract, substitute ${ }^{45}$.

Finding workable solutions will require not touching the T-zone to be considered much more seriously than it appears to be at the moment. This may be because it seems hard to imagine that a behaviour as trivial as this could make a difference in addressing such a huge crisis as a global pandemic. This could be an example of a yet-undocumented (to our knowledge) cognitive heuristic: what one might call the 'proportionality heuristic': a tendency to assume that the perceived magnitude of a solution to a problem should be proportionate to the magnitude of the problem.

\section{Conclusions}

In addition to isolation and social distancing measures, enactment of key personal protective behaviours is vital in order to reduce the transmission of SARS-CoV-2 and other respiratory viruses. Interventions to target individual behaviours such as these could potentially lead to substantial population-level effects ${ }^{46}$, and behavioural science models and methods can be used to develop and evaluate such interventions. There is currently a dearth of evidence on interventions to achieve these behaviour changes and an urgent need to rectify this. Given the urgency of the current situation, there may be merit in establishing an online hub for helping with the design of pragmatic evaluations, piloting of interventions, and rapid reporting of experiences and outcomes using a standardised approach.

Received: 7 April 2020; Accepted: 21 April 2020;

Published online: 6 May 2020 
Table 4 | Preliminary analysis of options for enacting behaviours to limit virus transmission

\section{General principles with regard to types of intervention}

1. Education: should focus on promoting understanding of the benefits of the behaviours and on how and when to enact them effectively, address concerns about the potential costs, and provide specific guidance on how to minimise adverse spill-over effects. It should take into account varying levels of educational level and varying circumstances.

2. Persuasion: should focus on generating a feeling of responsibility toward others in families and the community and a sense that the behaviours are valued by groups with which the target group identifies. It should promote concern and active engagement rather than anxiety and defensive avoidance.

3. Incentivization: should focus on use of social reward, including thanking people and praising them, to make people feel positive about having engaged in the behaviour.

4. Coercion: where threat of punishment is used, this must be accepted as appropriate by the community and applied in a way that is seen as equitable and proportionate. The contingencies should be clearly set out and explained. As far as possible any sanctions applied should be immediate (for example, on-the-spot fines). Use of social coercion should focus on the behaviour, not the person, and be delivered in a way that is perceived as supportive and just.

5. Training: should involve demonstrating the behaviour in full and component-by-component (including what to do and what not to do), provide a schedule for practice, and show common mistakes and how to rectify them.

6. Restriction: should involve setting clear and specific social rules around behaviours, with boundaries that are readily understood, explaining the rationale and providing specific examples of what is and is not acceptable.

7. Environmental restructuring: should involve redesigning indoor and outdoor spaces, include environmental cues that promote the behaviour, ensure that people have the material resources they need to enact the behaviours whenever required, create or provide access to physical spaces that support the behaviour, ensure that people have financial resources to sustain themselves and their families if they enact the behaviour, and ensure that social and organisational rules and norms are supportive of the behaviour.

8. Modelling: should show people with whom the target group identifies enacting the behaviour in ways that are culturally appropriate and realistic to achieve.

9. Enablement: should include a range of interventions for improving capability, such as therapeutic interventions to address mental health barriers to adherence, as well as interventions to extinguish or build habits and create if-then rules.

\section{Behaviour}

Only cough or sneeze into tissues that are safely disposed of

Wear face masks as appropriate

Maintain physical distance

Wash or disinfect hands under defined conditions

Disinfect surfaces and objects

Do not touch the T-zone unless one has just thoroughly washed one's hands

Social distancing (staying at home except under specifically defined circumstances)

Isolation of people who have symptoms or who are highly vulnerable

\section{Analysis}

Education, training, modelling and enablement to improve capability, delivered through mass media, social media and online videos to show how to do this effectively and build habits, including ensuring that people always carry tissues with them.

Education, persuasion and modelling to improve capability, delivered through mass media and social media to help people wear face masks correctly in appropriate settings and ensure that they use them and dispose of them or disinfect them effectively. Training using online videos or delivered in person where practicable.

Education, persuasion and modelling delivered through mass media and social media, to motivate people to keep a specified distance where possible, and to encourage people to use social incentives to motivate other people they interact with to do the same. Supportive social coercion to discourage unnecessary proximity. Local restrictions regarding access to areas such as shops and parts of shops. Environmental restructuring to increase access to safe spaces and markings to show safe distances in areas such as queues.

Education, training and modelling delivered through mass media and key workers to ensure understanding of when it is necessary and how it should be done, using what cleaning agents. Environmental restructuring to ensure that hand sanitisers or washing facilities are available for people entering settings such as shops, workplaces and educational facilities where they touch objects. Enablement provided through online platforms to support people to set up personal if-then rules and habits.

Education, training and modelling delivered through online platforms to promote effective routines for cleaning surfaces and objects, especially in public spaces. Environmental restructuring to ensure ready access to appropriate cleaning agents.

Education and persuasion using mass media and social media to ensure that people understand the importance of not touching parts of their face if there is any chance they have touched a contaminated surface or object. Training and enablement using online platforms and in-person support to help people extinguish the habit and build conflicting habits, and to cope with facial itches without touching with a hand or part of the hand that may be contaminated.

Education, persuasion, incentivization, coercion, restriction and modelling are all needed to ensure strong motivation to enact the behaviour in the face of strong competing motivation. The messaging has to be precise and seen as personally relevant and include examples to counter exceptionalism. Coercive measures and restrictions need to take into account people's need for a sense of agency. Environmental restructuring and enablement are particularly important to minimise negative spill-over effects and exacerbation of inequality. This must include financial and material resources and psychological support for people suffering from distress and mental health problems.

Education to ensure understanding of how and why to implement and sustain isolation and how to achieve it in challenging domestic settings. Environmental restructuring to ensure continued access to financial and material resources. Enablement provided by key workers to provide tailored support to people in maintaining daily functioning and mental health. 


\section{References}

1. Bradley, D.T., Mansouri, M.A., Kee, F. \& Garcia, L.M.T. A systems approach to preventing and responding to COVID-19. EClinicalMedicine https://doi. org/10.1016/j.eclinm.2020.100325 (2020).

2. Lunn, P. et al. Using behavioural science to help fight the coronavirus. $J$ Behav. Pub. Admin. 3, https://doi.org/10.30636/jbpa.31.147 (2020)

3. Scientific Pandemic Influenza behaviour Advisory Committee (SPI-B). The role of behavioural science in the coronavirus outbreak. https://assets. publishing.service.gov.uk/government/uploads/system/uploads/attachment_ data/file/873732/07-role-of-behavioural-science-in-the-coronavirus-outbreak pdf (SAGE, 2020).

4. Behavioural Science and Disease Prevention Taskforce. Behavioural science and disease prevention: psychological guidance. https:/www.bps.org.uk/sites/ www.bps.org.uk/files/Policy/Policy\%20-\%20Files/Behavioural\%20science $\% 20$ and $\% 20$ disease $\% 20$ prevention $\% 20$-\%20Psychological\%20guidance $\% 20$ for $\% 20$ optimising\%20policies\%20and\%20communication.pdf (British Psychological Society, 2020).

5. Van Bavel, J.J. et al. Using social and behavioural science to support COVID-19 pandemic response. Nat. Hum. Behav. https://doi.org/10.1038/ s41562-020-0884-Z (2020).

6. Taylor, S. The Psychology of Pandemics: Preparing for the Next Global Outbreak of Infectious Disease (Cambridge Scholars Publishing, 2019).

7. Shah, H. Global problems need social science. Nature 577, 295 (2020).

8. Lake, M. A. What we know so far: COVID-19 current clinical knowledge and research. Clin. Med. (Lond.) 20, 124-127 (2020).

9. NHS. NHS Advice on stopping spread of COVID-19 https://www.nhs.uk/ conditions/coronavirus-covid-19/ (2020).

10. Lu, J. et al. COVID-19 outbreak associated with air conditioning in restaurant, Guangzhou, China, 2020. Emerg. Infect. Dis. https://doi. org/10.3201/eid2607.200764 (2020).

11. World Health Organisation. Modes of transmission of virus causing COVID-19: implications for IPC precaution recommendations. https://www.who.int/ news-room/commentaries/detail/modes-of-transmission-of-virus-causing-covid 19-implications-for-ipc-precaution-recommendations (WHO, 2020).

12. Lewis, D. Is the coronavirus airborne? Experts can't agree. Nature 580, 175 (2020)

13. Cowling, B. J. et al. Impact assessment of non-pharmaceutical interventions against COVID-19 and influenza in Hong Kong: an observational study. Lancet Public Health https://doi.org/10.1016/S2468-2667(20)30090-6 (2020).

14. Alegado, S. Global Cost of Coronavirus May Reach \$4.1 Trillion, ADB Says. Bloomberg https://www.bloomberg.com/news/articles/2020-04-03/ global-cost-of-coronavirus-could-reach-4-1-trillion-adb-says (2 April 2020).

15. Brooks, S. K. et al. The psychological impact of quarantine and how to reduce it: rapid review of the evidence. Lancet 395, 912-920 (2020).

16. Feng, S. et al. Rational use of face masks in the COVID-19 pandemic. Lancet Respir. Med. S2213-2600(20)30134-X (2020).

17. Service, R. You may be able to spread coronavirus just by breathing, new report finds. Science https://www.sciencemag.org/news/2020/04/you-may-be-ablespread-coronavirus-just-breathing-new-report-finds\# (2 April 2020).

18. Bourouiba, L. Turbulent gas clouds and respiratory pathogen emissions: potential implications for reducing transmission of COVID-19. J. Am. Med. Assoc. https://doi.org/10.1001/jama.2020.4756 (2020).

19. Boone, S. A. \& Gerba, C. P. Significance of fomites in the spread of respiratory and enteric viral disease. Appl. Environ. Microbiol. 73, 1687-1696 (2007)

20. van Doremalen, N. et al. Aerosol and surface stability of SARS-CoV-2 as compared with SARS-CoV-1. N. Engl. J. Med. 382, 1564-1567 (2020).

21. National Health Service. Advice for everyone: Coronavirus. (COVID-19) https://www.nhs.uk/conditions/coronavirus-covid-19/ (2020).

22. Australian Department of Health. How to protect yourself and others from coronavirus (COVID-19). https://www.health.gov.au/news/health-alerts/ novel-coronavirus-2019-ncov-health-alert/ how-to-protect-yourself-and-others-from-coronavirus-covid-19 (2020).

23. Government of Canada. Coronavirus disease (COVID-19) prevention and risks. https://www.canada.ca/en/public-health/services/diseases/20 19-novel-coronavirus-infection/prevention-risks.html (2020).

24. Mbakaya, B. C., Lee, P. H. \& Lee, R. L. Hand hygiene intervention strategies to reduce diarrhoea and respiratory infections among schoolchildren in developing countries: a systematic review. Int. J. Environ. Res. Public Health 14, 371 (2017)

25. Doronina, O., Jones, D., Martello, M., Biron, A. \& Lavoie-Tremblay, M. A systematic review on the effectiveness of interventions to improve hand hygiene compliance of nurses in the hospital setting. J. Nurs. Scholarsh. 49, 143-152 (2017).

26. Schmidt, W.-P., Wloch, C., Biran, A., Curtis, V. \& Mangtani, P. Formative research on the feasibility of hygiene interventions for influenza control in UK primary schools. BMC Public Health 9, 390 (2009).

27. Chida, Y., Steptoe, A., Hirakawa, N., Sudo, N. \& Kubo, C. The effects of psychological intervention on atopic dermatitis. A systematic review and meta-analysis. Int. Arch. Allergy Immunol. 144, 1-9 (2007).
28. Hashimoto, K., Ogawa, Y., Takeshima, N. \& Furukawa, T. A. Psychological and educational interventions for atopic dermatitis in adults: A systematic review and meta-analysis. Behav. Change 34, 48-65 (2017).

29. Michie, S., Atkins, L. \& West, R. The Behaviour Change Wheel: A Guide to Designing Interventions (Silverback Publishing, 2014).

30. Michie, S., van Stralen, M. M. \& West, R. The behaviour change wheel: a new method for characterising and designing behaviour change interventions. Implement. Sci. 6, 42 (2011).

31. West, R., Michie, S., Atkins, L., Chadwick, P. \& Lorencatto, F. Achieving Behaviour Change: A Guide for Local Government and Partners (Public Health England, 2020).

32. Michie, S. et al. The behavior change technique taxonomy (v1) of 93 hierarchically clustered techniques: building an international consensus for the reporting of behavior change interventions. Ann. Behav. Med. 46, 81-95 (2013).

33. West, R. \& Brown, J. Theory of Addiction (Wiley, 2013).

34. Hornsey, M. J. Social identity theory and self-categorization theory: a historical review. Soc. Personal. Psychol. Compass 2, 204-222 (2008).

35. Davis, J.M. in Perspectives in Ethology (eds Bateson, P. P. G. \& Klopfer, P. H.) 43-72 (Springer, 1973).

36. Mayraz, G. Wishful thinking. SSRN https://doi.org/10.2139/ssrn.1955644 (2011)

37. Michie, S., West, R., Campbell, R., Brown, J. \& Gainforth, H. ABC of Behaviour Change Theories (Silverback Publishing, 2014).

38. Tversky, A. \& Kahneman, D. Advances in prospect theory: cumulative representation of uncertainty. J. Risk Uncertain. 5, 297-323 (1992).

39. Bouton, M.E. Learning and Behavior: A Contemporary Synthesis. (Sinauer Associates, 2007).

40. Kahneman, D. \& Miller, D. T. Norm theory: Comparing reality to its alternatives. Psychol. Rev. 93, 136-153 (1986).

41. Diefenbach, M. A. \& Leventhal, H. The common-sense model of illness representation: Theoretical and practical considerations. J. Soc. Distress Homeless 5, 11-38 (1996).

42. Michie, S., et al. Reducing SARS-CoV-2 transmission in the UK: a behavioural science approach to identifying options for increasing adherence to social distancing and shielding vulnerable people. Br. J. Health Psychol. (in the press).

43. Hallsworth, M. How to stop touching our faces in the wake of the Coronavirus. The Behavioural Insights Team https://www.bi.team/blogs/how-to-stoptouching-our-faces-in-the-wake-of-the-coronavirus/ (5 May 2020).

44. Clark, F., Sanders, K., Carlson, M., Blanche, E. \& Jackson, J. Synthesis of habit theory. OTJR (Thorofare, N.J.) 27, 7S-23S (2007).

45. West, R., Michie, S., Rubin, G.J. \& Amlot, R. Don't touch the T-zone. BMJO Opinion https://blogs.bmj.com/bmj/2020/04/03/dont-touch-the-t-zone-howto-block-a-key-pathway-to-infection-with-sars-cov-2/ (3 April 2020).

46. Sniehotta, F. F. et al. Complex systems and individual-level approaches to population health: a false dichotomy? Lancet. Public Health 2, e396-e397 (2017)

47. West, R. \& Michie, S. Routes of transmission of SARS-CoV-2 and behaviours to block it: a summary. Qeios https://doi.org/10.32388/F6M5CB (2020).

48. West, R. \& Michie, S. A brief introduction to the COM-B model of behaviour and the PRIME theory of motivation. Qeios https://www.qeios.com/read/ article/564 (2020).

49. Janis, I.L. \& Mann, L. Decision Making: A Psychological Analysis of Conflict, Choice, and Commitment (Free Press, 1977).

50. Greenwald, A. G. \& Ronis, D. L. Twenty years of cognitive dissonance: case study of the evolution of a theory. Psychol. Rev. 85, 53-57 (1978).

51. van den Bos, W. \& McClure, S. M. Towards a general model of temporal discounting. I. Exp. Anal. Behav. 99, 58-73 (2013).

52. McCrae, R. R. \& Costa Jr, P. T. The five-factor theory of personality. in Handbook of Personality: Theory and Research (eds John, O. P., Robins, R. W. \& Pervin, L. A.) 159-181 (Guilford Press, 2008).

\section{Acknowledgements}

G.J.R. and R.A. are affiliated with the National Institute for Health Research Health Protection Research Unit (NIHR HPRU) in Emergency Preparedness and Response at King's College London, in partnership with Public Health England (PHE), in collaboration with the University of East Anglia. The views expressed are those of the authors and not necessarily those of the NHS, the NIHR, the Department of Health and Social Care or Public Health England.

\section{Competing interests}

The authors declare no competing interests.

\section{Additional information}

Correspondence should be addressed to R.W.

Reprints and permissions information is available at www.nature.com/reprints.

Publisher's note Springer Nature remains neutral with regard to jurisdictional claims in published maps and institutional affiliations.

Editor recognition statement: Primary handling editor: Stavroula Kousta

(C) Springer Nature Limited 2020 